\title{
When visual transients impair tactile change detection: A novel case of crossmodal change blindness?
}

\author{
Alberto Gallace ${ }^{\mathrm{a}, \mathrm{b}, *}$, Malika Auvray ${ }^{\mathrm{a}, \mathrm{c}}$, Hong Z. Tan $^{\mathrm{d}}$, Charles Spence ${ }^{\mathrm{a}}$

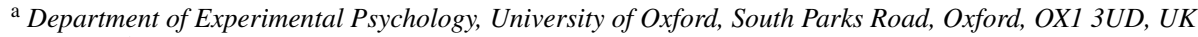 \\ ${ }^{\mathrm{b}}$ Dipartimento di Psicologia, Universita' degli Studi di Milano Bicocca, Milano, Italy \\ ${ }^{c}$ C.O.S.T.E.C.H, Université de Technologie de Compiègne, Compiègne, France \\ ${ }^{\mathrm{d}}$ Haptic Interface Research Laboratory, Purdue University, West Lafayette, USA
}

Received 26 September 2005; received in revised form 29 December 2005; accepted 5 January 2006

\begin{abstract}
The inability of people to detect changes between consecutively presented visual displays, when separated by a blank screen or distractor, is known as "change blindness". This phenomenon has recently been reported to occur within the auditory and tactile modalities as well. To date, however, only distractors presented within the same sensory modality as the change have been demonstrated to produce change blindness. In the present experiment, we studied whether tactile change blindness might also be elicited by the presentation of a visual mask. Participants made same versus different judgments regarding two successively presented displays composed of two to three vibrotactile stimuli. While change detection performance was near-perfect when the two displays were presented one directly after the other, participants failed to detect many of the changes between the tactile displays when they were separated by an empty temporal interval. Critically, performance deteriorated still further when the presentation of a local (i.e., a mudsplash) or global visual transient coincided with the onset of the second tactile pattern. Analysis of the results using signal detection theory revealed that this crossmodal effect reflected a genuine perceptual impairment.
\end{abstract}

(C) 2006 Elsevier Ireland Ltd. All rights reserved.

Keywords: Spatial representation; Multisensory; Attention; Body representation; Tactile information processing; Change blindness

Change blindness is the name given to the surprising inability of people to detect even obvious visual changes between consecutively-presented visual scenes. The phenomenon has been reported when irrelevant visual transients are presented between the to-be-compared displays (e.g., [10,20,27,30,32]). Many different stimuli/events have been shown to elicit visual change blindness when presented between the two to-becompared scenes: These include blank (or black) screens (e.g., [28]), eye blinks [26], saccades (e.g., [19]), movie cuts (e.g., [21]), and multiple discrete masking elements known as "mudsplashes" (e.g., [27]; see also [31,39]).

Change blindness has also been reported within the auditory modality (e.g., $[5,9,40]$ ) and more recently within touch [11-14]. In particular, recent studies conducted in this laboratory have demonstrated that participants frequently fail to detect the presence of positional and identity changes between simple consecutively-presented vibrotactile patterns (composed of two

\footnotetext{
* Corresponding author. Tel.: +44 1865 271380; fax: +44 1865310447.

E-mail address: alberto.gallace@psy.ox.ac.uk (A. Gallace).
}

or three discrete vibrotactile stimuli) presented over the body surface. Moreover, tactile change blindness has been demonstrated to be more pronounced when tactile distractors are superimposed on the display at the moment of change [13,14], in a similar fashion to that reported previously using the visual mudsplash paradigm [27].

Although tactile change blindness occurs for stimulus displays consisting of smaller numbers of tactile stimuli than for vision, the existence of a number of similarities between the change blindness phenomena in the two sensory modalities suggests the possibility of a common underlying mechanism (see $[11,14])$. Previously, we suggested that change blindness in vision as well as in touch might be related to the failure of a stimulus within a multisensory/amodal spatial representation where the change took place to reach awareness and/or draw spatial attention to itself.

In the present experiment, we specifically addressed the question of whether change blindness in different sensory modalities may have a common underlying substrate (perhaps related to the crossmodal nature of attention and/or the crossmodal representation of space; e.g., [34]). In particular, we postulated that if the 
principal cause of change blindness relates to the existence of a multisensory/amodal system used to process and integrate information, then the detection of tactile changes should be impaired not only when tactile transients are presented [11], but also when visual transients are presented at the time of the tactile change. Therefore, participants in the present study had to detect the presence of positional changes between two tactile displays (changes occurred on $50 \%$ of trials) during the concurrent presentation of a visual mask or of a visual mudsplash (see [27]).
Thirteen right-handed participants (seven males and six females) took part in this 25 min experiment as paid volunteers (mean age of 26.8 years, range of 19-33 years).

Participants sat on a chair for the duration of the experiment. The vibrotactile stimuli were presented by means of six resonant-type tactors (Part No: VBW32, Audiological Engineering Corp., Somerville, MA, USA) with $1.6 \mathrm{~cm} \times 2.4 \mathrm{~cm}$ vibrating surfaces. The tactors were placed on the participant's body on top of any clothing they happened to be wearing by

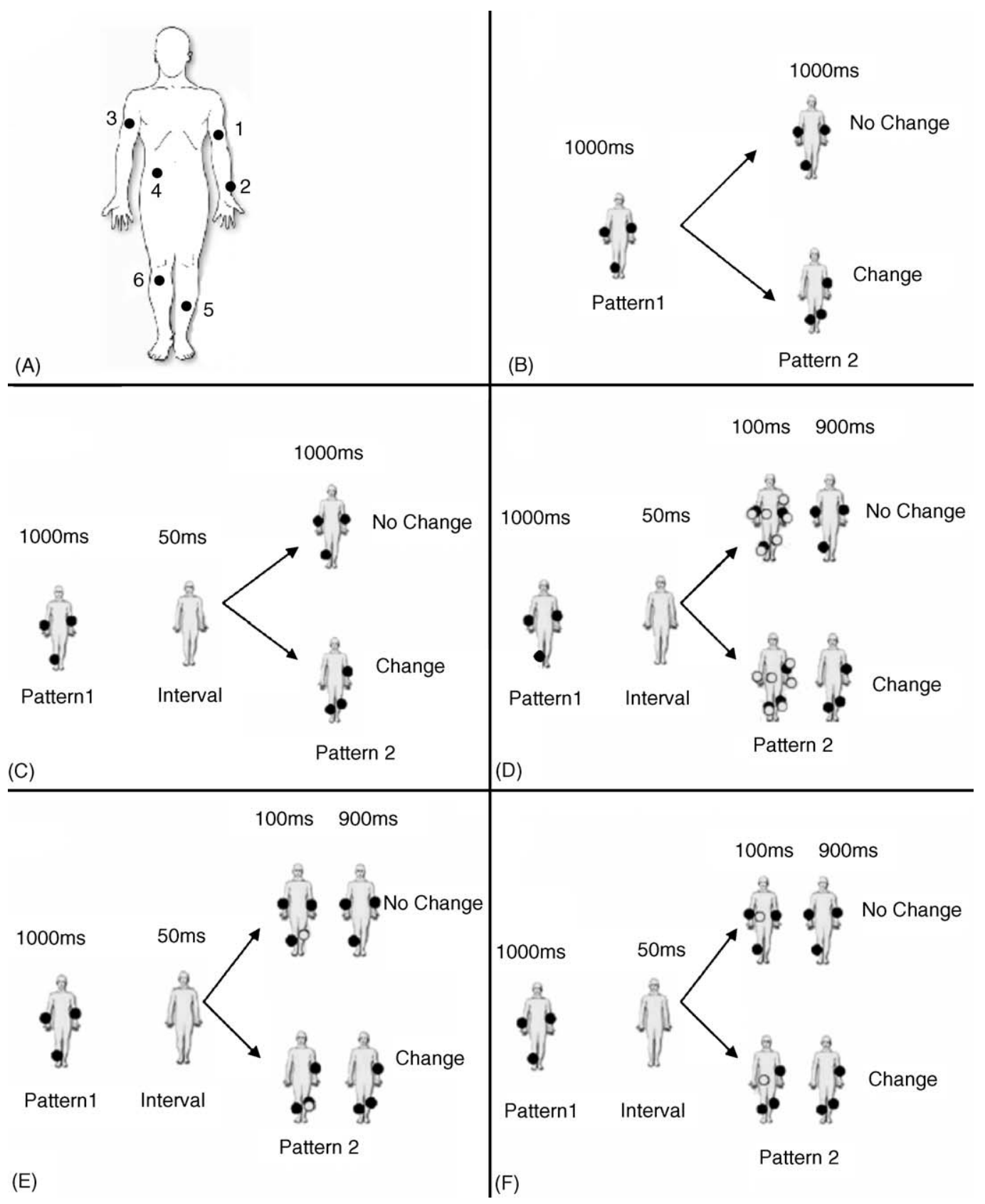

Fig. 1. (A) The positions on the body surface where the tactors and LEDs were placed. (1) left wrist; (2) just above the left elbow; (3) midway between the elbow and shoulder on the right arm; (4) on the waistline, to the right of the body midline; (5) just above the left ankle; and (6) midway between the ankle and knee on the right leg. Schematic illustration of the sequence of events presented in each trial of the experiment: (B) no interval; (C) empty interval; (D) visual mask; (E) coincident visual mudsplash; (F) non-coincident visual mudsplash. The numerical values shown above each figure indicate the duration (in ms) of the events concerned. Black discs represent vibrotactile stimuli and light grey discs (with black borders) visual stimuli. (Note that the visual stimuli were seen on the participant's body via mirror reflection.) 
means of Velcro strip belts. Green LEDs were mounted at the same position as each tactor but on the other side of the belts (see Fig. 1A, for the position of the tactors and LEDs on the body). The vibrators were driven by means of a custom-built 9channel amplifier circuit (Haptic Interface Laboratory, Purdue University, Indiana, USA) that drove each tactor independently at $290 \mathrm{~Hz}$ (close to its resonant frequency).

The intensity of each tactor was adjusted individually at the beginning of the experiment, so that each vibrotactile stimulus could be perceived clearly, and all of the tactile stimuli were perceived to be of similar intensity. The amplification levels for the tactors were kept at their individually-chosen levels throughout the experiment. White noise was presented over closed-ear headphones at $70 \mathrm{~dB}(\mathrm{~A})$ to mask any sounds made by the operation of the vibrotactile stimulators. A $65 \mathrm{~cm} \times 90 \mathrm{~cm}$ mirror was placed $100 \mathrm{~cm}$ in front of the participant (measured from the upper edge of the mirror to the participant's eyes). The ability of participants to correctly detect the visual stimuli presented from each body location was confirmed at the beginning of the experiment for each participant.

The stimuli consisted of two patterns, equiprobably composed of two or three vibrotactile stimuli. Both patterns were presented sequentially, and their duration was set at $1000 \mathrm{~ms}$ (i.e., we adopted the "one shot" procedure used in previous visual change blindness experiments; [6]). The positions of the stimuli were chosen randomly between all possible combinations of the six body sites stimulated. In the no change trials, the two vibratory patterns were identical. In the change condition, one of the vibrotactile stimuli composing the second pattern was presented from a different position. The intertrial interval (ITI) was set at $600 \mathrm{~ms}$.

The experiment was composed of five different blocks, presented in a randomized order between participants. In one block of trials, the vibrotactile stimuli were presented sequentially without any gap between them (no interval condition). In the other four blocks, the two patterns were always separated by a 50-ms empty interstimulus interval (cf. [11,14]). In one of these blocks, no visual stimuli were presented (empty interval condition). In the remaining three blocks, a visual transient was presented for $100 \mathrm{~ms}$ at the same time as the onset of the second vibrotactile pattern. In one block, all six LEDs were illuminated (visual mask condition). In the remaining two blocks, only one of the LEDs was illuminated (visual mudsplash conditions). The position of the LED that was illuminated was chosen randomly from amongst those body positions that were non-coincident with any of the tactile stimuli composing either of the two vibrotactile patterns (non-coincident visual mudsplash condition) in one block, and the body positions that were coincident with the position of the changed location (in the second tactile pattern) in the other block (coincident visual mudsplash condition; see Fig. 1B-F for a schematic illustration of the experimental conditions).

The participants pressed one of two keys on a computer keyboard depending upon whether or not the second vibrotactile pattern felt the same as the first. The trial was terminated if no response was made within $4 \mathrm{~s}$ of the offset of the second pattern. No feedback was given regarding the correctness of a participant's response. Participants were instructed to respond as accurately as possible. Eighty trials were presented in each experimental condition. In $50 \%$ of the trials, a positional change between the two patterns was presented, and in the remaining trials no change occurred. Each participant completed 400 trials in total.

Trials in which participants failed to make a response $(<1 \%$ of trials overall) were not analysed. The percentages of correct and erroneous change detection responses were used to calculate a measure of perceptual sensitivity $\left(d^{\prime}\right)$ and criterion $(\beta)$, for each block type using signal detection theory [23]. These measures were submitted to a repeated measures ANOVA with the factor of Block Type (five levels: no interval, empty interval, non-coincident visual mudsplash, coincident visual mudsplash, and visual mask). The analysis of the sensitivity data revealed a significant main effect of block type $[F(4,48)=13.5 ; p<.0001]$. A Duncan post-hoc test revealed significant differences between the no interval condition and all of the other block types (all $p<.05)$. The differences between the empty interval condition and each of the block types were also significant (all $p<.05$ ). None of the other differences (i.e., between any of the three visual masking conditions) reached statistical significance. The lowest $d^{\prime}$ value was observed in the visual mask condition and the highest value (signifying the most accurate performance) in the no interval condition (see Fig. 2A).

The analysis of the response bias data revealed a significant main effect of Block Type $[F(4,48)=3.72 ; p<.05]$. A Duncan post-hoc test revealed significant differences between the no interval condition and all of the other experimental conditions (all $p<.05$ ). None of the other differences were significant.
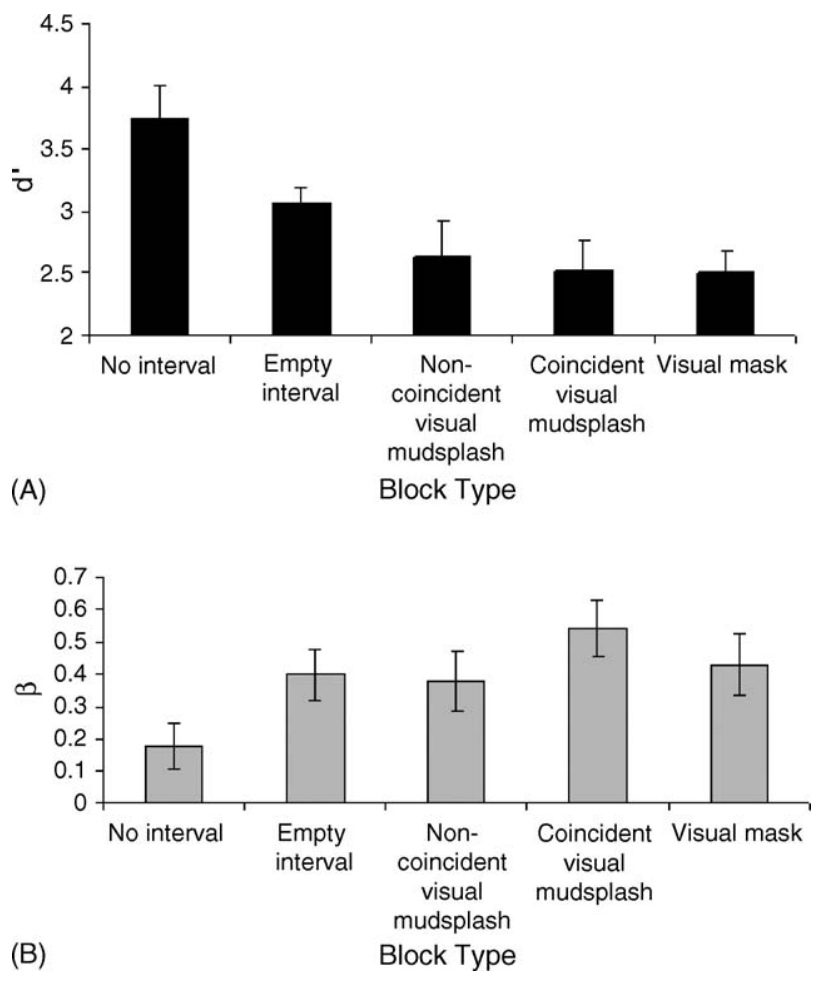

Fig. 2. Performance in each experimental block: (A) mean $d^{\prime}$ values; (B) mean $\beta$ values. Error bars represent the standard errors of the means. 
The highest $\beta$ value was observed in the non-coincident visual mudsplash condition and the lowest value in the no interval condition (see Fig. 2B). The significant effect reported for $\beta$ appears to be related to the near-perfect performance observed in the no interval block of trials (with $\beta$ close to zero indicating little response bias). Therefore, the differences in performance reported between the various different blocks of trials appear to be determined by a change in participants sensitivity rather than by a change in response bias. ${ }^{1}$

The results showed a significant difference in people's sensitivity between the condition in which the two tactile displays were temporally separated and the condition where they were presented without interruption on the body surface. This result confirms the existence of tactile change blindness when people have to detect the presence of a positional change between two sequentially-presented vibrotactile patterns $[11,14]$. More surprisingly, our results also demonstrate that the phenomenon can be elicited when a transient (or change) occurs simultaneously in a different sensory modality (here vision). Participants' sensitivity to detect the occurrence of a change between the two vibrotactile displays decreased (as compared to the empty interval condition) when a "visual" mask or distractor (mudsplash) coincided temporally with the tactile onset of the second display.

No significant performance differences were reported between the spatially-coincident and spatially non-coincident visual mudsplash conditions. At first glance, this result would appear to conflict with the results of a unimodal visual study of change blindness obtained by O'Regan et al. [27] using a mudsplash paradigm. They reported that when a single blackand-white textured rectangle (instead of multiple mudsplashes) briefly covered the location of the change at the moment of change in the display, participants were able to detect the presence of the change fairly accurately. However, the fact that: (a) the change in O'Regan et al.'s study was not a positional change but an onset or offset change (i.e., an object either being added to or removed from the display); (b) O'Regan et al. used a very different "unimodal" visual set-up; and (c) the visual and tactile stimuli were never actually spatially-coincident in the present

\footnotetext{
${ }^{1}$ We performed a second experiment $(N=12$ participants) in order to determine whether a visual mudsplash or mask would also impair tactile change detection performance when the visual stimuli were not presented on the participant's body. The methods were the same as in our main experiment with the following exceptions: (1) the visual stimuli were presented on a wall $2 \mathrm{~m}$ in front of the participant (the positions of the visual stimuli were the same as those adopted in the main experiment but linearly projected onto the wall, while maintaining similar visual angles between the stimuli); (2) in the mudsplash condition only non-coincident mudsplashes were used. The results of an ANOVA performed on the $\mathrm{d}^{\prime}$ data showed a significant effect of the experimental condition $[F(3,33)=3.82 ; p<.05]$. A Duncan post-hoc test revealed significant differences between the visual mask and no-interval conditions, and between the mudsplash and the no-interval conditions (all $p<.05$ ), but not between the other conditions. A borderline significant difference was found between the empty-interval and mudsplash condition $(p=.058)$. No significant differences were found on the analysis of the beta values $[F(3,33)<1 ;$ n.s.]. These results therefore show that visual stimuli no matter whether they are presented on the participant's body (i.e., in the same position as the vibrotactile stimuli) or not (i.e., when presented on the wall in front of the participant), can impair tactile change detection performance.
}

study, but were instead seen via mirror reflection, makes any direct comparison between the two experiments difficult.

The most important finding to emerge from the present experiment is the significant difference between all of the conditions in which a visual stimulus was presented at the time of the change (the mudsplash and mask conditions) and the condition in which an empty interval separated the presentation of the two tactile patterns. Given that in both conditions the duration of the temporal gap between the two patterns was the same $(50 \mathrm{~ms})$, the decrease in performance observed in the visual mask and mudsplash conditions can only be attributed to the presence of the visual transients interfering with tactile discrimination performance. This result clearly shows that tactile change blindness can be elicited by masking within the same sensory modality as the change [12], but also by "masking" presented in a different sensory modality. Such a crossmodal effect on change detection performance cannot, however, simply be attributed to some form of low-level sensory masking (cf. [17,33]), and must instead reflect some form of higher-order central masking (e.g., [1,4]; cf. [18]). Furthermore, it is also worth noting that the influence of the visual distractors on tactile change detection performance in the present study does not reflect response bias (as measured by $\beta$ ), but would instead appear to highlight a genuine perceptual effect (as indexed by the reduction in perceptual sensitivity, as measured by $d^{\prime}$ ).

We previously proposed that change blindness in vision as well as in touch might be related to people's lack of awareness of, and/or inability to direct attention to (or to be captured by), the spatial position where a change was represented (or occurred) within a multisensory/amodal representation $[11,14]$. This might be the result of competition between the representation of different concurrently stimulated positions [6], and/or of the limited availability of human information processing resources [42]. Wright et al. reported that visual change detection performance is influenced by the number of stimuli presented in a display (i.e., the higher the number of stimuli, the worse the performance). Interestingly, recent results from numerosity judgment studies obtained using multisensory stimulus presentation have shown that the system responsible for processing information regarding spatial location is limited multisensorially in terms of the number of stimuli that can access consciousness, draw attention to themselves, or else elicit a response, regardless of their sensory modality of presentation $[12,13]$. Consequently, it might be plausible that if change blindness reflects the failure of a stimulus (presented in a given spatial position where the change took place) to reach awareness and/or spatial attention to itself within a multisensory/amodal spatial representation, visual distractors should be expected to be effective in eliciting tactile change blindness (as demonstrated for tactile distractors; $[11,14]) .^{2}$ These are precisely the results obtained in the present

\footnotetext{
2 A further experiment was also conducted in order to investigate whether visual change blindness would be elicited by the presentation of tactile distractors. The methods were the same as our main experiment with the only difference being that the to-be-compared patterns were visual while the distractors were tactile. However, performance in this visual change blindness experiment was nearly errorless in all of the experimental conditions (i.e., when tactile distrac-
} 
study. Indeed, when concurrent transients are presented from different spatial positions, they compete for access to consciousness and/or to capture attention (see [6]; see also [24]; cf. [43]). Therefore, it would appear that in situations where unimodal transients are suppressed, and the number of positions stimulated (regardless of the sensory modality of that stimulation) exceeds a certain limit, change blindness may well be observed. Alternatively however, the possibility that change blindness may be elicited by the competition to elicit awareness/draw attention between the representations of stimuli irrespective of their spatial location cannot be ruled out on the basis of the present results. This important matter should be addressed in future research.

Interestingly, our results show that it is not only visual transients presented on the body itself but also visual stimuli presented at some distance from the participants $(2 \mathrm{~m})$ that can elicit tactile change blindness (see footnote 1). This result suggests that the multisensory/amodal representation in which the stimuli compete for accessing consciousness and/or drawing attention to themselves (cf. [7]) is not a body-based representation, but may instead constitute a more allocentric or environmental spatial representation.

Responses to a target in a given sensory modality (for example touch) are faster and more accurate when spatially-non predictive cues (in either the same or different sensory modality) are presented relatively close in space and time to the target (an effect that is often referred to as "crossmodal exogenous spatial attentional cuing"; e.g., see [35], for a recent review). Although the contribution of multisensory integration versus covert spatial attention to the behavioural effects that have been observed in such cuing studies is still a matter of some debate (e.g., [25]; see Spence et al. [35,36], for recent discussion of this issue), it appears clear that stimuli presented in one sensory modality interact with the spatial processing of stimuli presented in another sensory modality, regardless of their task-relevance. The results of the experiment reported here are consistent with this observation but here using a completely different experimental paradigm (i.e., one involving pattern perception and change detection), thereby strengthening the claim that a multisensory representation might be responsible for spatial processing and the awareness of spatial information (e.g., [34]; see also [41]).

The results of the present experiment show for the first time that tactile change blindness can be elicited by the presentation of visual transients, therefore strengthening the claim that change blindness may in fact be a multisensory phenomenon related to the competition between transients in terms of capturing attention and/or eliciting awareness.

\footnotetext{
tors were presented, when an empty interval was presented between the displays, and when the two visual patterns were presented without any interval between them). This null effect is probably related to the small number of visual stimuli used in the display ( 2 or 3 , i.e., the same number used in the main tactile change detection experiment). Note that this number of stimuli falls below the visual "subitizing" limit (i.e., the limit on people's ability to report errorlessly the number of items presented in a visual display when this number does not exceed the limit of 4-5 units), as demonstrated by studies of visual numerosity judgments (e.g., [2]). By contrast, people appear unable to subitize tactile stimuli presented on their body [12].
}

\section{Acknowledgements}

A.G. was supported by a grant from the Universita' di Milano Bicocca, Italy. H.Z.T. and C.S. were supported by a Network Grant from the Oxford McDonnell-Pew Centre for Cognitive Neuroscience. M.A. was supported by a Lavoisier fellowship from the Ministère des Affaires Entrangères, France. Correspondence regarding this article should be addressed to Alberto Gallace, Room B121, Department of Experimental Psychology, University of Oxford, Oxford, UK, OX1 3UD. E-mail: alberto.gallace@psy.ox.ac.uk.

\section{References}

[1] E.A. Alluisi, B.B. Morgan Jr., G.R. Hawkes, Masking of cutaneous sensations in multiple stimulus presentations, Percept. Motor Skills 20 (1965) 39-45.

[2] J. Atkinson, F.W. Campbell, M.R. Francis, The magic number $4 \pm 0$ : a new look at visual numerosity judgements, Perception 5 (1976) 327-334.

[4] J. Brehaut, J.T. Enns, V. Di Lollo, Visual masking plays two roles in the attentional blink, Percept. Psychophys. 61 (1999) 1436-1448.

[5] J.S. Chan, C. Spence, Change deafness: an auditory analogue of visual change blindness? Acta Psychologica, submitted for publication.

[6] G.G. Cole, R.W. Kentridge, A.R.H. Gellatly, C.A. Heywood, Detectability of onsets versus offsets in the change detection paradigm, J. Vision 3 (2003) 22-31.

[7] R. Desimone, J. Duncan, Neural mechanisms of selective visual attention, Annu. Rev. Neurosci. 18 (1995) 193-222.

[9] R. Eramudugolla, D.R.F. Irvine, K.I. McAnally, R.L. Martin, J.B. Mattingley, Directed attention eliminates 'change deafness' in complex auditory scenes, Curr. Biol. 21 (2005) 1108-1113.

[10] R.S. French, The discrimination of dot patterns as a function of number and average separation of dots, J. Exp. Psychol. 46 (1953) 1-9.

[11] A. Gallace, H.Z. Tan, C. Spence, Failure to detect tactile change: a tactile equivalent to the change blindness phenomenon. Psychonomic Bull. Rev., in press.

[12] A. Gallace, H.Z. Tan, C. Spence, Numerosity judgments for tactile stimuli distributed over the body surface, Perception, in press.

[13] A. Gallace, H.Z. Tan, C. Spence, Multisensory numerosity judgments. Percept. Psychophys., submitted for publication.

[14] A. Gallace, H.Z. Tan, C. Spence, Tactile change blindness for onset/offset events following tactile mask and mudsplashes. Percept. Psychophys., submitted for publication.

[17] G.A. Gescheider, R.K. Niblette, Cross-modality masking for touch and hearing, J. Exp. Psychol. 74 (1967) 313-320.

[18] B. Giesbrecht, W.F. Bischof, A. Kingstone, Visual masking during the attentional blink: tests of the object substitution hypothesis, J. Exp. Psychol.: Hum. Percept. Perform. 29 (2003) 238-258.

[19] J. Grimes, On the failure to detect changes in scenes across saccades, in: K. Atkins (Ed.), Percept.: Vancouver Studies in Cognitive Science, vol. 5, 1996, pp. 89-109.

[20] J. Hochberg, In the mind's eye, in: R.N. Haber (Ed.), Contemporary Theory and Research in Visual Perception, Holt, Rhinehart \& Winston, New York, 1968, pp. 309-331.

[21] D.T. Levin, D.J. Simons, Failure to detect changes to attended objects in motion pictures, Psychonomic Bull. Rev. 4 (1997) 501-506.

[23] N.A. Macmillan, C.D. Creelman, Detection theory: a user's guide, second ed., Lawrence Erlbaum Associates, New York, 2004.

[24] R. Martin-Emerson, A.F. Kramer, Offset transients modulate attentional capture by sudden onsets, Percept. Psychophys. 59 (1997) 739-751.

[25] J.J. McDonald, W.A. Teder-Sälejärvi, L.M. Ward, Multisensory integration and crossmodal attention effects in the human brain, Science 292 (2001) 1791.

[26] J.K. O'Regan, H. Deubel, J.J. Clark, R.A. Rensink, Picture changes during blinks: looking without seeing and seeing without looking, Visual Cogn. 7 (2000) 191-212. 
[27] J.K. O'Regan, R.A. Rensink, J.J. Clark, Change-blindness as a result of "mudsplashes", Nature 398 (1999) 34.

[28] R.A. Rensink, J.K. O'Regan, J.J. Clark, To see or not to see: the need of attention to perceive changes in scenes, Psychol. Sci. 8 (1997) 368-373.

[30] D.J. Simons, In sight, out of mind: when object representations fail, Psychol. Sci. 7 (1996) 301-305.

[31] D.J. Simons, S.L. Franconeri, R.L. Reimer, Change blindness in the absence of a visual disruption, Perception 29 (2000) 1143-1154.

[32] D.J. Simons, R.A. Rensink, Change blindness: past, present, and future, Trends Cognitive Sci. 9 (2005) 16-20.

[33] S. Soto-Faraco, C. Spence, K. Fairbank, A. Kingstone, A.P. Hillstrom, K. Shapiro, A crossmodal attentional blink between vision and touch, Psychon. Bull. Rev. 9 (2002) 731-738.

[34] C. Spence, J. Driver (Eds.), Crossmodal Space and Crossmodal Attention, Oxford University Press, Oxford, UK, 2004.

[35] C. Spence, J. McDonald, J. Driver, Exogenous spatial-cueing studies of human cross-modal attention and multisensory integration, in: C. Spence,
J. Driver (Eds.), Crossmodal Space and Crossmodal Attention, Oxford University Press, Oxford, UK, 2004, pp. 277-320.

[36] C. Spence, F. Pavani, J. Driver, Spatial constraints on visual-tactile crossmodal distractor congruency effects, Cognitive, Affective, Behav. Neurosci. 4 (2004) 148-169.

[39] M. Turatto, S. Bettella, C. Umiltà, B. Bridgeman, Perceptual conditions necessary to induce change blindness, Visual Cogn. 10 (2003) 233-255.

[40] M.S. Vitevitch, Change deafness: the inability to detect changes between two voices, J. Exp. Psychol.: Hum. Percept. Perform. 29 (2003) 333342.

[41] L.M. Ward, J.A. McDonald, N. Golestani, Cross-modal control of attention shifts, in: R. Wright (Ed.), Visual Attention, Oxford University Press, New York, 1998, pp. 232-268.

[42] M. Wright, A. Green, S. Baker, Limitations for change detection in multiple Gabor targets, Visual Cogn. 7 (2000) 237-252.

[43] V.A.F. Lamme, Why visual attention and awareness are different, Trends Cognitive Sci. 7 (2003) 12-18. 